\title{
A tryptophan-rich peptide acts as a transcription activation domain
}

\author{
Chen-Huan Lin, Grace Lin, Chia-Pei Chang, Chien-Chia Wang ${ }^{*}$
}

\begin{abstract}
Background: Eukaryotic transcription activators normally consist of a sequence-specific DNA-binding domain (DBD) and a transcription activation domain (AD). While many sequence patterns and motifs have been defined for DBDs, ADs do not share easily recognizable motifs or structures.

Results: We report herein that the N-terminal domain of yeast valyl-tRNA synthetase can function as an AD when fused to a DNA-binding protein, LexA, and turn on reporter genes with distinct LexA-responsive promoters. The transcriptional activity was mainly attributed to a five-residue peptide, WYDWW, near the C-terminus of the N domain. Remarkably, the pentapeptide per se retained much of the transcriptional activity. Mutations which substituted tryptophan residues for both of the non-tryptophan residues in the pentapeptide (resulting in $W_{5}$ ) significantly enhanced its activity ( 1.8-fold), while mutations which substituted aromatic residues with alanine residues severely impaired its activity. Accordingly, a much more active peptide, pentatryptophan $\left(W_{7}\right)$, was produced, which elicited $\sim 3$-fold higher activity than that of the native pentapeptide and the $\mathrm{N}$ domain. Further study indicated that $\mathrm{W}_{7}$ mediates transcription activation through interacting with the general transcription factor, TFIIB.
\end{abstract}

Conclusions: Since $W_{7}$ shares no sequence homology or features with any known transcription activators, it may represent a novel class of $A D$.

\section{Background}

Eukaryotic transcriptional activators that stimulate transcription initiation of a particular set of target genes usually consist of a sequence-specific DNA-binding domain (DBD) and a transcription activation domain (AD). The DBD targets these activators to a specific location in the promoter region of a gene, and the AD mediates transcription initiation by recruiting gene-specific factors, chromatin-remodeling factors, mediator complexes, and general transcription factors [1,2]. DBDs are classified into several distinct patterns or motifs according to their sequences and structural similarities. In contrast, ADs do not share significant sequence homologies, and therefore no specific motif has been defined. Despite this, several classes of ADs with distinctive sequence features were identified, including acidic activators [3], glutamine-rich activators [4], and proline-rich activators [5]. While an increasing number of transcriptional activators

\footnotetext{
* Correspondence: dukewang@cc.ncu.edu.tw

Department of Life Science, National Central University, Jung-li 32001, Taiwan
}

( 2010 Lin et al; licensee BioMed Central Ltd. This is an Open Access article distributed under the terms of the Creative Commons Attribution License (http://creativecommons.org/licenses/by/2.0), which permits unrestricted use, distribution, and reproduction in any medium, provided the original work is properly cited. have been identified, the direct targets or interacting partners of most transcriptional activators and the detailed mechanisms through which these interacting partners induce transcription initiation remain largely unknown. To date, only a few protein factors have been explicitly identified as direct targets of transcriptional activators, including the TATA box-binding protein (TBP), TFIIB, TFIIH, and a few others [6].

One of the earliest and best-studied models for transcriptional regulation in eukaryotes is that of yeast Gal4, which is involved in regulating galactose metabolism in response to changes in the concentration of the carbohydrate [2,7]. The Gal4 system (also known as the GAL regulon) consists of four structural (GAL1, GAL2, GAL7, and GAL10) and three regulatory (GAL3, GAL4, and GAL80) genes. Protein products of the structural genes are required for transport and metabolism of galactose, and protein products of the regulatory genes control expression of the structural genes. Gal4 is an acidic transcriptional activator comprised of two functionally independent domains: an $\mathrm{N}$-terminal sequence-specific 
DBD and a C-terminal AD. Induction of the Gal4responsive genes by galactose is mediated through the specific binding of the Gal4-DBD to an upstream activating sequence in their promoter regions and subsequent recruitment of the general transcription apparatus by the Gal4-AD. Under non-inducing conditions in the absence of galactose, Gal4 activity is repressed by the interaction of Gal80 with the Gal4-AD [8,9]. Under inducing conditions when galactose is present, the repressor, Gal80, is taken away by Gal3 from the Gal4$\mathrm{AD}$, which is then able to recruit the transcription machinery and initiate transcription [10].

In prokaryotes, there are typically 20 aminoacyl-tRNA synthetases, one for each amino acid [11-14]. In eukaryotes, protein synthesis occurs in the cytoplasm, and also in organelles, such as mitochondria and chloroplasts [15]. Thus, eukaryotes, such as yeast, commonly have two genes that encode distinct sets of proteins for each aminoacylation activity, one localized in the cytoplasm and the other in the mitochondria. However, in some cases, the cytoplasmic and mitochondrial forms of a tRNA synthetase with a given amino acid specificity are encoded by the same nuclear gene through alternative initiation of translation, examples of which include ALA1 (which codes for alanyl-tRNA synthetase) [16], GRS1 (which codes for glycyl-tRNA synthetase) [17], HTS1 (which codes for histidyl-tRNA synthetase) [18], and VAS1 (which codes for valyl-tRNA synthetase (ValRS)) [19]. Because the two isoforms are essentially generated from the same open reading frame, they have the same polypeptide sequence, except for a short sequence attached to the amino-terminus of the mitochondrial precursor that functions as a mitochondrial targeting signal. As a consequence, the two isoforms cannot be substituted for each other in vivo.

Many eukaryotic cytoplasmic tRNA synthetases contain an amino- or carboxyl-terminal polypeptide extension, which is absent from their bacterial counterparts [20]. These extensions are generally rich in lysine residues and capable of non-specifically binding to RNA. A well-studied example is the appended domain (Ad) of yeast glutaminyl-tRNA synthetase (GlnRS), which binds unfractionated yeast tRNAs, single-stranded RNA, and pseudoknot RNA with comparable affinities, with $K_{\mathrm{d}}$ values of around $0.6 \mu \mathrm{M}[21,22]$. Many other examples were also identified, such as the carboxyl-terminal domain of rice methionyl-tRNA synthetase [23] and the amino-terminal domain of mammalian lysyl-tRNA synthetase [24]. In addition to RNA binding, the Ads of some tRNA synthetases were found to participate in protein-protein interactions, such as those of yeast glutamyl- and methionyl-tRNA synthetases (GluRS and MetRS) [25] and mammalian ValRS [26]. However, more generally, the exact role of the Ad in the biological functions of this family of enzymes remains elusive. Recently, it was found that many of these Ads contain one or several classical nuclear localization signals [27] which are believed to play a role in the nuclear importation of these otherwise "cytoplasmic" proteins. Even more exciting are the findings that several tRNA synthetases from both prokaryotes and eukaryotes take part in functions unrelated to aminoacylation, including roles in mitochondrial RNA splicing, transcriptional and translational regulation, cytokine-like activity, and amino acid biosynthesis $[28,29]$.

As with many known yeast tRNA synthetases, the cytoplasmic form of yeast ValRS also contains an amino-terminal polypeptide extension. While the Ad of mammalian ValRS was shown to interact with the four subunits of the elongation factor, EF- $1 \mathrm{H}$, to form a high-molecular-weight complex [26], relatively little is known about the biological function of its yeast counterpart. Our earlier studies suggested that the Ad of yeast ValRS (residues 1 98) possesses non-specific tRNAbinding activity (with a $K_{\mathrm{d}}$ of $\sim 2 \mu \mathrm{M}$ ) that significantly contributes to tRNA binding and aminoacylation activities of the enzyme [30,31]. We report herein that the $\mathrm{N}$-terminal domain (residues 1 135) of yeast ValRS can act as an $\mathrm{AD}$ when fused to a sequence-specific $\mathrm{DBD}$, and this transcriptional activity is mainly attributable to a tryptophan-rich peptide (WYDWW) within the N domain. Using this pentapeptide as a reference structure, a much more active peptide, heptatryptophan $\left(\mathrm{W}_{7}\right)$, was consequently devised. Furthermore, $\mathrm{W}_{7}$ stimulated transcription initiation via interaction with TFIIB, a general transcription factor. It is our hope that information obtained in this study will advance our understanding of the biochemical properties of ADs in general, and also provide new insights into the mechanisms of transcription activation in particular.

\section{Results}

\section{Identification of an activation peptide}

In addition to tRNA binding, we wondered whether the $\mathrm{N}$-terminal domain of yeast ValRS possesses another function. To explore this possibility, the $\mathrm{N}$-terminal domain (residues 1 135) was cloned by fusion to the DNA-binding protein, LexA, and used as bait to screen a yeast library for interacting partners. Note that the $\mathrm{N}$-terminal sequence used in this assay was 37 residues longer than the Ad (residues 1 98) used in previous studies $[30,31]$ to ensure that all of the sequence elements that might be important for interactions were included. After all, the exact length of the Ad has yet to be clearly defined. The DNA sequence encoding the Nterminal domain was PCR-amplified and cloned as a lexA fusion into pGilda (carrying an HIS3 marker) as described in "Materials and Methods", and the resulting 
construct was cotransformed with the reporter plasmid, p8oplacZ (carrying a URA3 marker), into the EGY48 yeast strain. To rule out the possibility that the bait hybrid protein was itself an autoactivator, the cotransformants were first tested on selection medium (minimal medium containing X-gal but lacking uracil, histidine, and leucine) in the absence of any prey hybrid protein. Unexpectedly, the LexA-N domain fusion by itself could turn on the LEU2 and lacZ reporter genes; the cotransformants grew robustly on the X-gal agar plate and turned blue (Figure 1A, number 3). This result suggests that the bait hybrid protein per se is a transcription activator, and the $\mathrm{N}$ domain of yeast ValRS acts as an $\mathrm{AD}$.

It was noted that the operator sequences in the reporter genes $L E U 2$ and lacZ were not identical, and only fusions that could turn on both reporter genes were considered positive in this assay. To determine which segment of the $\mathrm{N}$ domain actually accounted for this transcriptional activity, various segments of the domain were individually fused to LexA, and their transcriptional activities were tested. As shown in Figure 1A, segments containing residues 98 115, 101 115, 104 115, 107 115, and 111 115 were active in the transcriptional assay (numbers 4 8). In contrast, segments consisting of residues 113 115, 98 112, and 98 109 had no detectable activity (numbers 9 11). Thus, the segment containing residues 111 115 (WYDWW) is essential and sufficient for this activity (number 8 ).

Quantitative assays of the $\beta$-gal activity (encoded by the reporter gene carried on p8oplacZ) further showed that among these active peptides, the segments containing residues $98 \sim 115,101 \sim 115$, and 104 115 had the highest activities $(\sim 1.5$-fold relative to that of the positive control); the segment containing residues 107 115 had medium activity $(\sim 0.8$-fold relative to that of the positive control); and the segments containing residues $1 \sim 135$ and 111 115 had the lowest activities ( 0.5-fold relative to that of the positive control) (Figure 1B). As expected, functionally inactive peptides had no detectable $\beta$-gal activity in the assays (Figure $1 \mathrm{~B}$, numbers 9 11). To check whether all of these LexA fusion constructs were properly expressed in the reporter yeast strain, the expression profiles of these constructs were analyzed by Western blotting using an anti-LexA antibody. As shown in Figure 1C, all of the LexA fusion proteins were stably expressed by the constructs, with only minor variations in protein levels, suggesting that the negative phenotype observed on the X-gal plate for the functionally inactive peptides was not caused by severe protein degradation or insufficient protein synthesis (Figure 1A, numbers 9 11). pLexA-Pos (Gal4 fused to LexA) and pGilda respectively served as positive and negative controls in the assays.

\section{Repetition of the pentapeptide sequence enhanced activity}

While the pentapeptide, WYDWW, can function as an $\mathrm{AD}$, its activity was relatively low, only $\sim 50 \%$ of that of the positive control and $\sim 30 \%$ of that of the segment containing residues $98 \sim 115$ of the $\mathrm{N}$ domain (Figure 1B, numbers 1, 4, and 8). To enhance its activity, two or three tandem repeats of the pentapeptide sequence were cloned, and the activities of the resulting constructs were tested. As shown in Figure $2 \mathrm{~A}$ and $2 \mathrm{~B}$, duplication of the pentapeptide, resulting in (WYDWW $)_{2}$, strongly enhanced the activity $(\sim 3$-fold increase relative to that of a single pentapeptide) (compare numbers 3 and 4). However, an additional replication of the sequence, resulting in (WYDWW $)_{3}$, did not further enhance the

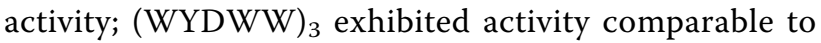

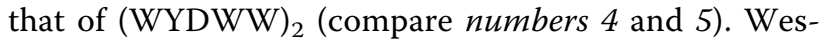
tern blotting assays showed that these constructs expressed similar levels of LexA fusion proteins. Thus, changes in the transcriptional activity of these fusion constructs did not result from different protein expression levels (Figure 2C, numbers 3 5).

\section{$\mathrm{W}_{5}$ had activity higher than that of WYDWW}

To examine which amino acid residues of the pentapeptide are critical for its activity, the residues were mutated to alanine residues, and the activities of the resulting constructs were tested. As shown in Figure 3, mutation of the first two amino acid residues (WY) to AA, the last two residues (WW) to AA, or the middle residue (D) to $A$ or $K$ drastically reduced the activities (compare numbers $3,4,5,6$, and 8 ). In contrast, mutation of the middle residue (D) to $\mathrm{W}$ had little effect on the activity (compare numbers 3 and 7). Moreover, mutation of the second residue $(\mathrm{Y})$ to $\mathrm{W}$ significantly enhanced the activity $(\sim 1.8$-fold) (compare numbers 3 and 9). Thus, it appears that $\mathrm{W}$ is preferred in all positions of the pentapeptide for activity. A Western blot analysis showed that these mutations had only minor effects on the expression levels of the fusion proteins (Figure 3C).

To gain further insights, both of the non-W residues in the pentapeptide were mutated to $\mathrm{W}$ residues (resulting in $\mathrm{W}_{5}$ ), and the activity of the resulting construct was tested. As expected, $\mathrm{W}_{5}$ had an activity $\sim 1.8$-fold higher than that of the native pentapeptide (Figure 4A, $\mathrm{B}$, numbers 3 and 4). Most amazingly, inserting two more $W$ residues into $W_{5}$, yielding $W_{7}$, further enhanced the activity ( 1.8-fold) (compare numbers 4 and 5 ). That is, the activity of $W_{7}$ was $\sim 3$-fold higher than that of the native pentapeptide (compare number 3 and 5). However, the activity of $\mathrm{W}_{9}$ was almost equivalent to that of $\mathrm{W}_{5}$ (numbers 4 and 6). Thus, $\mathrm{W}_{7}$ appeared to be the strongest AD among those tested. 


\begin{tabular}{|c|c|c|c|}
\hline & $\underline{\text { Construct }}$ & $\underline{\mathbf{A D}}$ & $\underline{\text { X-gal }}$ \\
\hline (1) & pLexA-Pos & Gal4-AD & prosests \\
\hline (2) & pGilda & None & was of \\
\hline (3) & pCW279 & --ALKAYNPANVESSWYDWW- - & खित्र \\
\hline (4) & pGL45 & ALKAYNPANVESSWYDWW & andes \\
\hline (5) & pCHL15 & AYNPANVESSWYDWW & a 204 \\
\hline (6) & pCHL16 & PANVESSWYDWW & boveng \\
\hline (7) & pGL165 & VESSWYDWW & 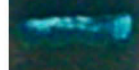 \\
\hline$(8)$ & pGL177 & WYDWW & Find \\
\hline (9) & pCHL49 & DWW & \\
\hline (10) & pCHL20 & ALKAYNPANVESSWY & \\
\hline (11) & pCHL21 & ALKAYNPANVES & serien \\
\hline
\end{tabular}

B

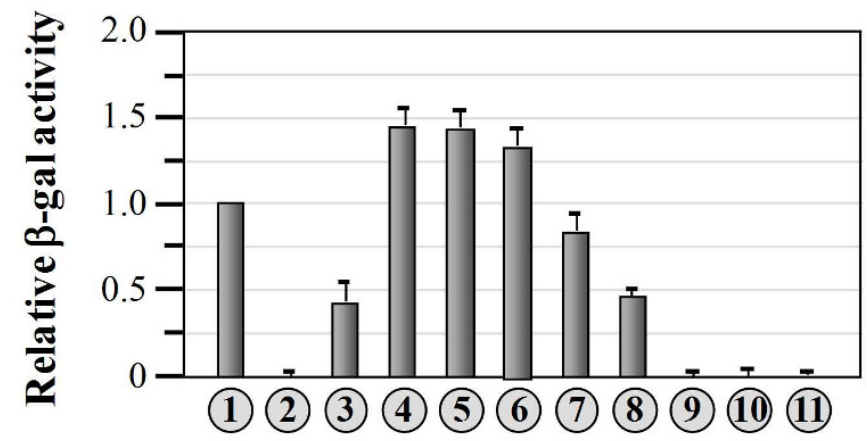

C
(2)
(3) (4)
(5) 6
(7)
(8)
(9) (10) (11)

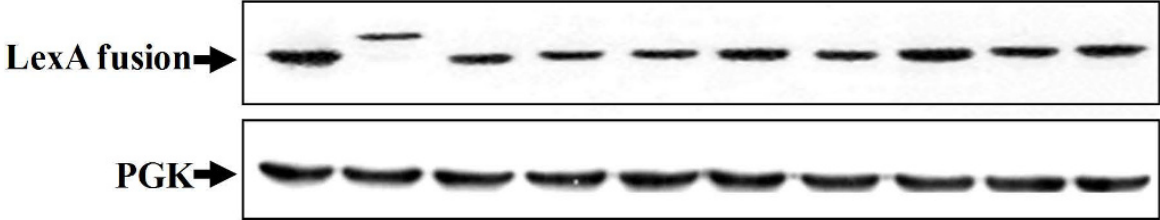

Figure 1 Assays of the transcriptional activity of the $\mathbf{N}$ domain of yeast valyl-tRNA synthetase. (A) Transcriptional assays. Various segments of the $\mathrm{N}$ domain of yeast ValRS were expressed as fusions to the specific DNA-binding protein, LexA, and the abilities of the resulting fusions to turn on the reporter genes (LEU2 and lacZ) which are controlled by distinct LexA-responsive promoters were tested. Activation of the reporter genes was indicated by the ability of the transformants to grow on selection medium lacking histidine, uracil, and leucine and to turn blue. (B) Quantitative assays of $\beta$-gal activity. (C) Western blot analysis of the expressions of LexA fusion proteins. Upper panel, LexA fusion protein; lower panel, phosphoglycerate kinase (PGK) (as a loading control). The numbers (circled) in $\mathbf{A}, \mathbf{B}$, and $\mathbf{C}$ represent the constructs shown in $\mathbf{A}$. 


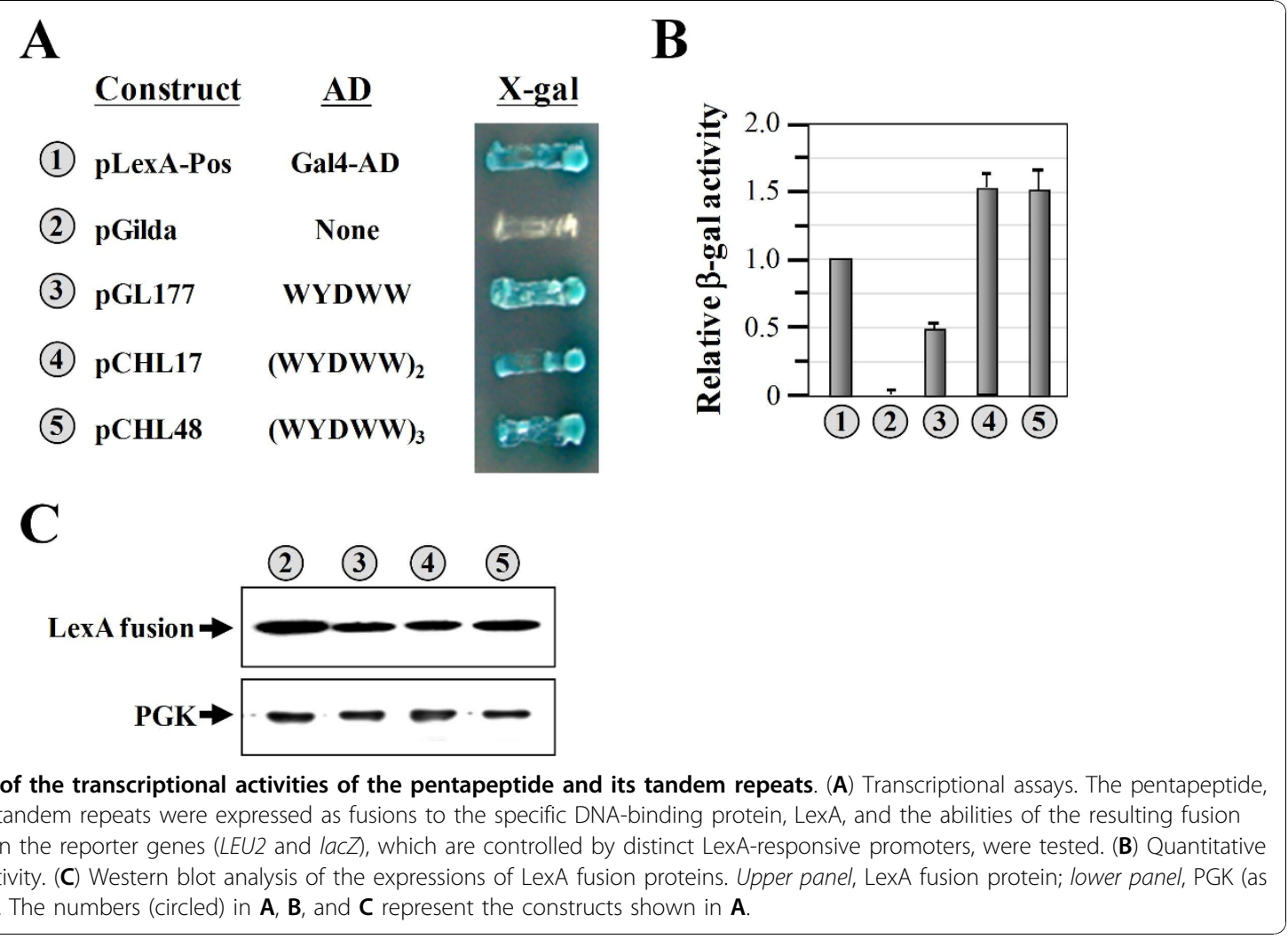

To investigate whether the transcriptional activities of these peptides were attributable to their hydrophobic property, $\mathrm{W}_{7}$ was mutated to $\mathrm{F}_{7}$, and the activity of the resulting construct was tested. As shown in Figure 4A and $4 \mathrm{~B}$, changing $\mathrm{W}_{7}$ to $\mathrm{F}_{7}$ resulted in a functionally inactive peptide that failed to turn on the reporter genes (numbers 5 and 7). With respect to protein expression, these fusion constructs expressed considerably different levels of proteins. LexA-W $W_{5}$ and LexA-W $-W_{9}$ produced the highest protein expression levels (Figure 4C, numbers 4 and 6); LexA-WYDWW and LexA-W 7 had medium protein expression levels (numbers 3 and 5); and LexA$\mathrm{F}_{7}$ had the lowest protein expression level (number 7).

To further investigate whether the different protein expression levels observed herein were caused by different protein stabilities in vivo, a cycloheximide-chase assay (or degradation assay) was carried out on the representative constructs, LexA-WYDWW, LexA$\mathrm{W}_{7}$, and LexA-F $\mathrm{F}_{7}$. To exclude the interference of transcription activation, the host cell used for the assay was INVSc1, instead of the reporter yeast strain, EGY48. As shown in Figure 4E, these fusion proteins had conspicuously distinct turnover rates. LexA-WYDWW was much more stable than LexA-W $W_{7}$, and LexA-W 7 was much more stable than LexA-F 7 . LexA- $\mathrm{F}_{7}$ had a short half-life (of $<15 \mathrm{~min}$ ) and was degraded in vivo at a much faster speed than the other two fusion proteins tested. It remains to be seen whether this attribute actually accounted for the negative phenotype of LexA- $\mathrm{F}_{7}$ in the transcriptional assay (Figure 4A, B). Another interesting finding was the discovery that while LexA- $\mathrm{W}_{7}$ had the highest transcriptional activity among these three constructs, it did not have the highest protein stability or expression level (Figure 4, numbers 3, 5, and 7). Thus, it appears that there were no direct correlations between transcriptional activity and protein expression levels in these instances. Figure 4D shows that similar levels of cDNA products were generated from these fusion constructs, suggesting that the sequences encoding WYDWW, $\mathrm{W}_{7}$, and $\mathrm{F}_{7}$ did not compromise the stability of the specific lexA mRNAs in vivo.

\section{$W_{7}$ is a non-promoter-specific $A D$}

We next tested whether the transcriptional activities of $\mathrm{W}_{7}$ and (WYDWW) 2 were promoter-specific, and whether they were affected by the DBD used. To this end, $\mathrm{W}_{7}$ and (WYDWW) ${ }_{2}$ were assayed in a Gal4-based system, where the AD was fused in-frame to the Gal4DBD cloned in pGBKT7 (which carries a TRP1 marker), and the reporter genes used were HIS3 and MEL1 (which encodes $\alpha$-galactosidase) under the control of two completely heterologous Gal4-responsive upstream activating sequences and promoter elements, GAL1 and $M E L 1$, respectively. As shown in Figure 5, both Gal4- 


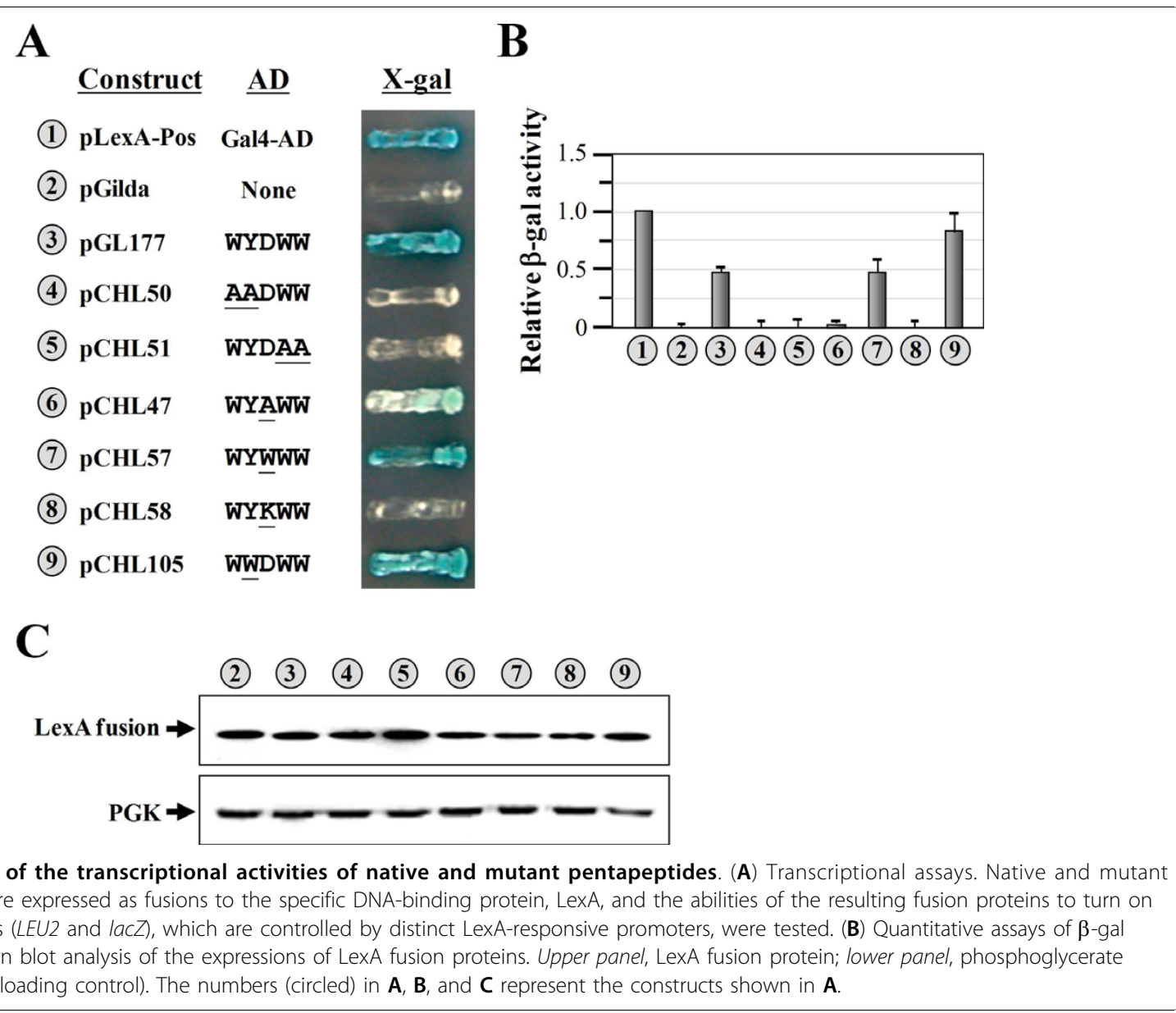

DBD fusion proteins turned on the reporter genes; transformants carrying either of these two fusion constructs (Gal4-DBD-W 7 or Gal4-DBD-(WYDWW) ${ }_{2}$ ) grew robustly and turned blue on selection medium containing $\mathrm{X}-\alpha$-gal but lacking tryptophan and histidine (Figure $5 \mathrm{~A}$, numbers 3 and 4), suggesting that both of these peptides acted as ADs in Gal4-DBD fusion proteins. Thus, the transcriptional activities of these two peptides were non-promoter-specific and were operational in both LexA- and Gal4-DBD-responsive reporter genes.

Quantitatively, these two fusion proteins had transcriptional activities $\sim 2.5$-fold lower than that of the positive control (Figure 5B, numbers 1, 3, and 4). This is not surprising, considering the fact that the positive control was a wild-type GAL4 gene. Western blot assays showed that these two Gal4-DBD fusion constructs had protein expression levels much lower than that of the Gal4-DBD alone (Figure 5C, numbers 2 4). Whether the ADs destabilized the Gal4-DBD fusion proteins and whether the relatively poor transcriptional activity of these two fusion constructss was caused by a lower level of protein expression are yet to be determined. However, regardless of the diverse protein expression levels, these results clearly demonstrate the ability of these two peptides to function as ADs in the Gal4-based system.

\section{$W_{7}$ acts as an $A D$ in a two-hybrid system}

So far, we have shown that $\mathrm{W}_{7}$ and (WYDWW) ${ }_{2}$ can act as ADs when directly fused to a sequence-specific DBD such as LexA and Gal4-DBD (Figures 2, 4, 5). The question arose as to whether these two short peptides can act as efficiently when they are physically separated from the DBD. To answer this question, DNA sequences encoding these two peptides were individually cloned into pGADT7-T (a prey fusion vector encoding the Gal4-AD-T fusion protein) to replace the sequence encoding the Gal4-AD, and the transcriptional activities of the resulting constructs were tested following the protocols devised for the yeast two-hybrid system. As shown in Figure 6, murine p53 (as a part of the Gal4DBD-53 fusion) specifically interacted with the SV40 large T-antigen (as a part of the Gal4-AD-T fusion) and turned on the reporter genes, HIS3 and MEL1 (number 1 , serving as the positive control). In contrast, human lamin C (as a part of the Gal4-DBD-Lam fusion) did not 


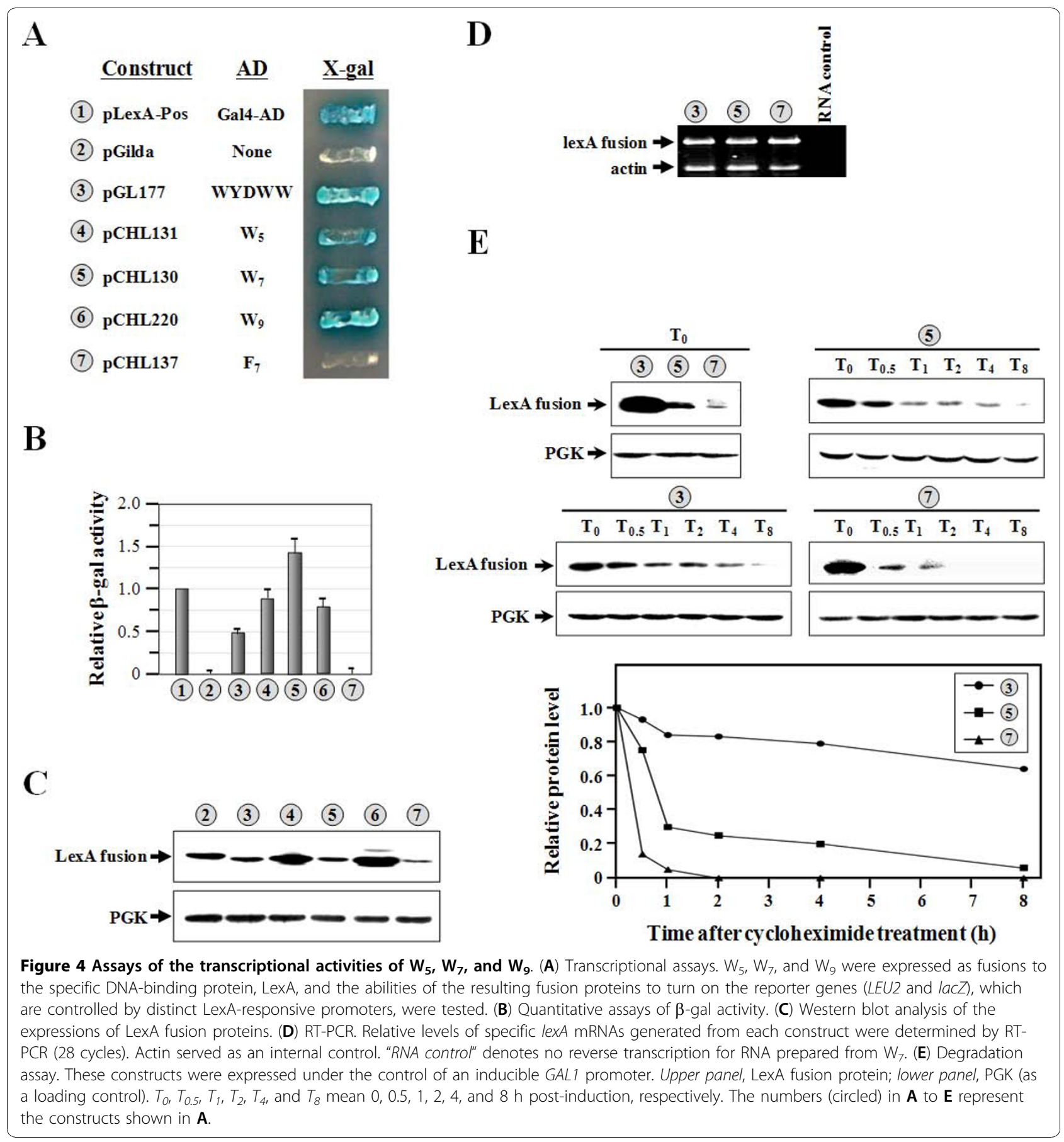

interact with the large T-antigen and therefore failed to turn on the reporter genes (number 2, serving as the negative control). Interestingly, both $\mathrm{W}_{7}-\mathrm{T}$ and (WYDWW $)_{2}$-T fusions turned on the reporter genes when acting in concert with the Gal4-DBD-53 fusion (numbers 3 and 5), but failed to do so when acting in concert with the Gal4-DBD-Lam fusion numbers 4 and 6). This observation provides strong evidence that both $\mathrm{W}_{7}$ and (WYDWW) 2 can act as ADs in a traditional two-hybrid system, albeit with efficiencies poorer than that of Gal4-AD (Figure 6B, numbers 1, 3, and 5).

\section{$\mathrm{W}_{7}$ mediates transcription activation through interacting} with TFIIB

To date, only a few protein factors have unambiguously been identified as direct targets of transcriptional activators, including TBP, TFIIB, TFIIH, and a few others [6]. We wondered whether $\mathrm{W}_{7}$ also stimulates transcription 


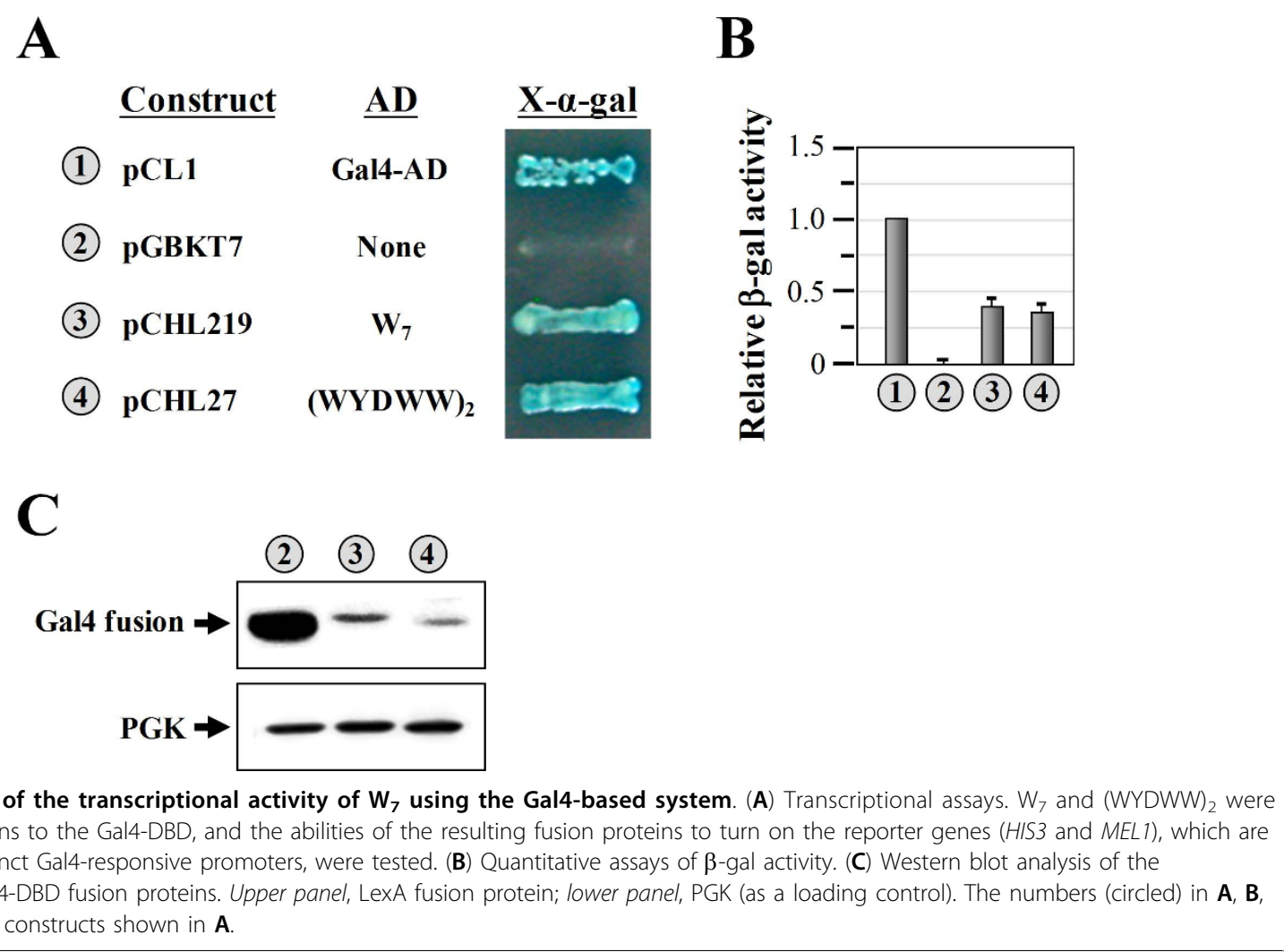

initiation through interaction with one of these protein factors. Pursuant to this objective, TBP, TFIIB, and TFIIH were individually cloned into pGilda (a bait fusion vector encoding the LexA fusion protein), and then their interaction with $\mathrm{W}_{7}$ (as a part of the $\mathrm{B} 42-\mathrm{W}_{7}$ fusion protein) was tested using a yeast two-hybrid system. LexA and B42 respectively served as the DBD and AD in this assay. As shown in Figure 7, all of these LexA fusion proteins were well expressed in the test yeast strain EGY48 (Figure 7B), with LexA-TFIIH having a lower expression level. In the absence of $\mathrm{W}_{7}$, none of these fusion constructs per se could simultaneously turn on the designated reporter genes, LEU2 and lacZ (Figure 7A). However, in the presence of $\mathrm{W}_{7}$, TFIIB, but not TBP or TFIIH, could turn on both reporter genes (Figure $7 \mathrm{C}$ ). This result strongly suggests that $\mathrm{W}_{7}$ specifically interacts with TFIIB.

\section{Discussion}

We report herein that the $\mathrm{N}$-terminal domain of yeast ValRS acts as an AD when fused to a sequence-specific DBD, LexA (Figure 1). However, there is no direct evidence so far showing that yeast ValRS actually functions as a transcription activator in the nucleus. More experiments are currently underway to validate this possibility. Despite this uncertain aspect, a core sequence element accounting for this activity was mapped to a region near the $\mathrm{C}$-terminus of the $\mathrm{N}$ domain. This core sequence element contains only five amino acid residues: WYDWW (N-terminal residues 111 115) (Figure 1). Interestingly, the core sequence element per se retained much of the transcriptional activity and could be stably expressed as a fusion to the DBD in yeast (Figure 1). A mutagenesis study indicated that $\mathrm{W}$ residues in the pentapeptide were crucial for its activity. Even the Y residue, which also possesses an aromatic ring in its side chain, was not as supportive of the activity as were the $\mathrm{W}$ residues (Figure 3). As a result, the native pentapeptide acted less efficiently $(\sim 1.8$-fold $)$ than did WWDWW and WWWWW as an AD (Figures 3,4). Using this core sequence element as a reference, a much-stronger activation peptide, $\mathrm{W}_{7}$, was successfully produced. $\mathrm{W}_{7}$ had $\sim 3$-fold higher transcriptional activity than those of the native pentapeptide and the $\mathrm{N}$ domain (Figures 1,4). While we cannot rule out the possibility that $\mathrm{W}_{7}$ somehow altered the affinity or specificity for the lexA operators of the LexA fusion protein, $\mathrm{W}_{7}$ has to be capable of interacting with one of the components of the transcription apparatus to stimulate transcription of the reporter genes. To our knowledge, $\mathrm{W}_{7}$ is so far the smallest $\mathrm{AD}$ that retains strong transcriptional activity. With its small size, $W_{7}$ is unlikely to interfere with the folding of fused proteins or to occlude the normal 
Two-hybrid construct

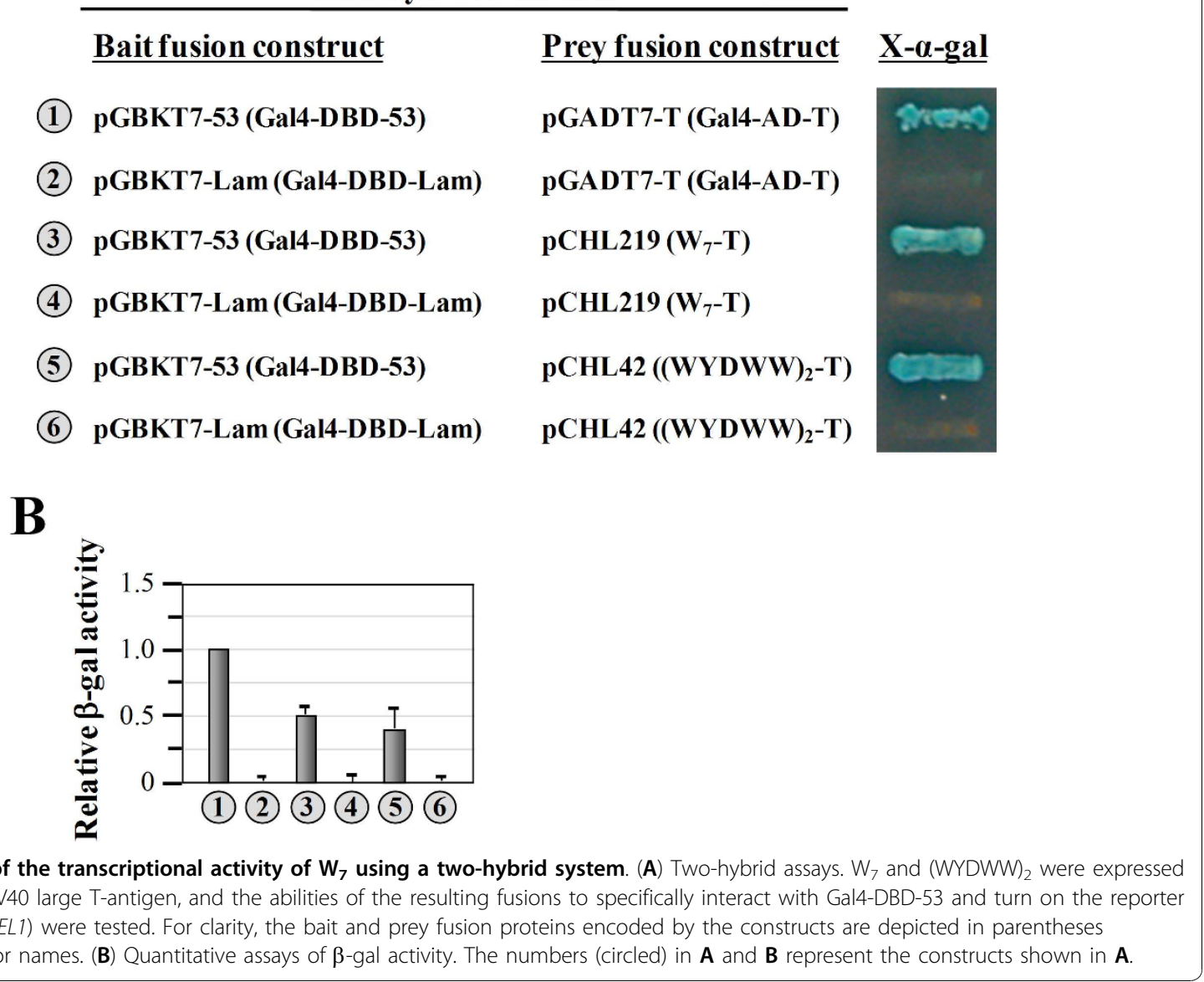

site of interaction. In addition, $\mathrm{W}_{7}$ can significantly reduce the overall size of the prey fusion construct, and therefore has the potential to enhance the efficiency of plasmid transformation, which is a long-standing issue when screening a large library for novel interacting partners. Similar to the reasons that the $\mathrm{His}_{6}$ tag is widely accepted and used for protein purification, $\mathrm{W}_{7}$ may be an excellent replacement for the Gal4-AD or B42 in two-hybrid screening as an AD.

A preliminary study suggested that the direct target of $\mathrm{W}_{7}$ may be the general transcription factor, TFIIB (Figure 7), which was previously shown to be one of the interacting partners of the acidic transcription activator, Gal4 [10]. It is believed that recruitment of TFIIB and others by Gal4 leads to assembly of the transcription apparatus at the promoter site and subsequent transcription initiation [7]. However, sequence alignment between these two ADs (Gal4-AD and $\mathrm{W}_{7}$ ) showed little sequence homology. Thus, Gal4-AD and $\mathrm{W}_{7}$ may interact with different sites of TFIIB or through a different mechanism. Alternatively, a certain portion of Gal4 may fold into a three-dimensional structure that displays a hydrophobic feature similar to that of $\mathrm{W}_{7}$. In any case, it is interesting to find that both of these ADs mediate transcription activation through interacting with TFIIB. Thus, the minute $\mathrm{AD}, \mathrm{W}_{7}$, might turn out to be an interesting paradigm for further mechanistic studies of transcription activation.

\section{Conclusions}

A short peptide containing seven consecutive tryptophan residues $\left(\mathrm{W}_{7}\right)$ can function as an activation domain when fused to a DNA-binding protein, LexA, and turn on reporter genes with distinct LexA-responsive promoters. Like the activation domain of Gal4, $\mathrm{W}_{7}$ mediates transcription activation through interacting with the general transcription factor, TFIIB.

\section{Methods}

\section{Construction of various LexA and Gal4-DBD fusion} constructs

To clone the DNA sequence encoding the $\mathrm{N}$ domain of yeast ValRS (base pairs +1 to +405 ) into pGilda and 


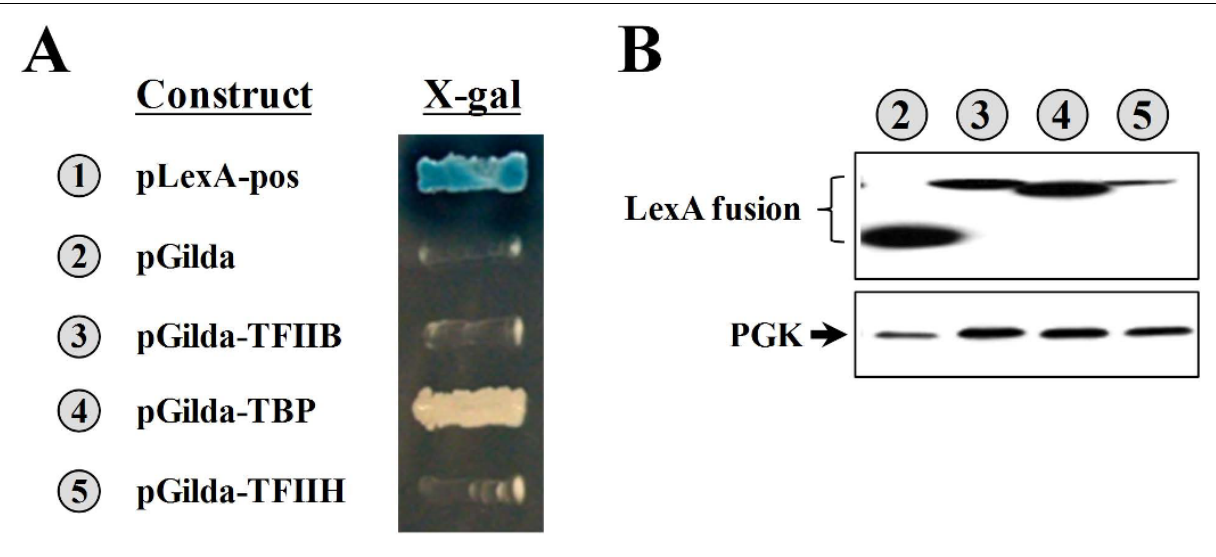

Two-hybrid construct

\begin{tabular}{|c|c|c|}
\hline Bait fusion construct & Prey fusion construct & $\underline{X-g a l}$ \\
\hline pLexA-53 (LexA-53) & pB42AD-T (B42-T) & Q... \\
\hline pGilda (LexA) & pB42AD-W $7\left(\right.$ B42-W $\left._{7}\right)$ & 82.4 \\
\hline pGilda-TFIIB (LexA-TFIIB) & pB42AD-W $\left(\mathrm{B}_{7}-\mathrm{W}_{7}\right)$ & 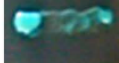 \\
\hline pGilda-TBP (LexA-TBP) & pB42AD-W $7\left(\right.$ B42-W $\left._{7}\right)$ & Eas \\
\hline pGilda-TFIIH (LexA-TFIIH) & pB42AD-W $7\left(\right.$ B42-W $\left._{7}\right)$ & arces \\
\hline
\end{tabular}

Figure 7 Identification of the interacting partners of $\mathbf{W}_{\mathbf{7}}$ using a two-hybrid system. (A) Transcriptional assay. TBP, TFIIB, and TFIIH were expressed as fusions to LexA, and the abilities of the resulting fusions to turn on the reporter genes (LEU2 and lacZ) were tested. (B) Western blot analysis of the expressions of LexA fusion proteins. Upper panel, LexA fusion protein; lower panel, PGK (as a loading control). The numbers (circled) in $\mathbf{A}$ and $\mathbf{B}$ denote the constructs shown in $\mathbf{A}$. (C) Two-hybrid assays. TBP, TFIIB, and TFIIH were expressed as fusions to LexA, and the abilities of the resulting fusions to specifically interact with $W_{7}$ and turn on the reporter genes (LEU2 and lacZ) were tested. For clarity, the bait and prey fusion proteins encoded by the constructs are depicted in parentheses following the vector names.

pGBKT7 (BD Biosciences Clontech, Palo Alto, CA) for the transcriptional assays, a set of primers complementary to nucleotides -15 to +15 and +390 to +420 of $V A S 1$ were respectively used to amplify the DNA fragment using a plasmid-borne VAS1 gene as a template. The forward primer contained an EcoRI site, and the reverse primer contained an XhoI site. The 405-bp PCR fragment was first digested with EcoRI and XhoI and then cloned into appropriate sites in pGilda or pGBKT7. To clone shorter fragments of the $\mathrm{N}$ domain, such as those coding for amino acid residues $98 \sim 115$ and $111 \sim 115$, or their derivatives, such as those coding for $\mathrm{W}_{5}, \mathrm{~W}_{7}$, and $\mathrm{W}_{9}$, into these two vectors, a pair of complementary oligonucleotides coding for the desired sequences were annealed and then cloned into the EcoRI-XhoI sites of these vectors. Sequences of the cloned DNA fragments were verified by DNA sequencing.

\section{Transcriptional assay}

This assay essentially followed the protocol of a yeast two-hybrid assay provided by the manufacturer (BD Biosciences Clontech), except that the prey cloned in pB42AD was not used. Briefly, the gene of interest was first cloned as a lexA fusion into pGilda (carrying an HIS3 marker) as mentioned above. The lexA fusion construct was then co-transformed with the reporter plasmid, p8oplacZ (carrying a URA3 marker and a lacZ reporter gene under the control of eight copies of the LexA operator and the minimal GAL1 promoter), into the EGY48 yeast strain (carrying an LEU2 reporter gene under the control of six copies of the LexA operator and the minimal LEU2 promoter), and the resulting cotransformants were selected on minimal medium lacking uracil and histidine. A single colony of the cotransformants that grew on the selection medium was picked and streaked on an agar plate containing X-gal 
but lacking uracil, histidine, and leucine. The streaked colony could not grow on the X-gal plate and turn blue unless the LexA fusion protein had turned on both reporter genes (LEU2 and lacZ). Note that the DNAbinding protein, LexA, alone could not activate transcription of the LexA-responsive reporter genes, but could do so when fused to an AD.

Alternatively, the transcriptional activity of the $\mathrm{N}$ domain was tested using a Gal4-based system (BD Biosciences Clontech), in which the DNA sequence encoding the $\mathrm{N}$ domain was fused in-frame to the sequence encoding Gal4-DBD cloned in pGBKT7 (carrying a TRP1 marker), and the resulting construct was then transformed into the AH109 reporter yeast strain and tested for its ability to turn on the reporter genes, HIS3 and MEL1. HIS3 was under the control of the GAL1 upstream activating sequence and a minimal promoter containing the GAL1 TATA box. The expression of $M E L 1$ was controlled by the intact MEL1 promoter, including the $M E L 1$ upstream activating sequence and MEL1 minimal promoter. The transformants could not grow and turn blue on selection medium containing X$\alpha$-gal but lacking tryptophan and histidine unless the Gal4-DBD fusion protein had turned on both reporter genes.

\section{Western blot analysis}

Protein expression patterns of the LexA fusions were determined by a chemiluminescence-based Western blot analysis. The transformants used in the transcriptional assays were grown in selection medium lacking uracil and histidine. Total protein extracts were prepared from the transformants with a buffer containing $50 \mathrm{mM}$ Tris (pH 7.4), $150 \mathrm{mM} \mathrm{NaCl}, 0.5 \%$ sodium dodecylsulfate (SDS), $0.5 \%$ Triton X-100, $10 \mathrm{mM}$ ethylenediaminetetraacetic acid, and $1 \mathrm{mM}$ phenylmethanesulfonyl fluoride. Aliquots of the protein extracts $(\sim 40 \mu \mathrm{g})$ were loaded onto a mini gel $(8 \times 10 \mathrm{~cm})$ containing $10 \%$ polyacrylamide and electrophoresed at $100 \mathrm{~V}$ for $\sim 2 \mathrm{~h}$. Following electrophoresis, the resolved proteins were transferred to a polyvinylidene difluoride (PVDF) membrane using a semi-dry transfer device. The membrane was probed with a horseradish peroxidase (HRP)-conjugated anti-LexA antibody (Invitrogen, Carlsbad, CA) and then exposed to $x$-ray film following the addition of appropriate substrates. The protein expression patterns of the Gal4-DBD fusions were determined following a similar protocol.

\section{$\beta$-Galactosidase ( $\beta$-Gal) assay}

Yeast cells were pelleted by centrifugation at $12,000 \times g$ for $30 \mathrm{~s}$ and resuspended in $100 \mu \mathrm{l}$ of breaking buffer (100 mM Tris- $\mathrm{HCl}$ (pH 8.0), $1 \mathrm{mM}$ dithiothreitol, $10 \%$ glycerol, and $2 \mathrm{mM}$ phenylmethanesulfonyl fluoride) and $100 \mu \mathrm{l}$ of beads. Cells were then lysed at $4{ }^{\circ} \mathrm{C}$ using a bead beater, followed by centrifugation at $12,000 \times g$ for $2 \mathrm{~min}$. Aliquots of the supernatants $(25 \sim 250 \mu \mathrm{g})$ were diluted to $0.8 \mathrm{ml}$ with $\mathrm{Z}$ buffer $\left(60 \mathrm{mM} \mathrm{Na}_{2} \mathrm{HPO}_{4}\right.$, $40 \mathrm{mM} \mathrm{NaH}_{2} \mathrm{PO}_{4}, 10 \mathrm{mM} \mathrm{KCl}, 1 \mathrm{mM} \mathrm{MgSO}_{4}$, and 50 mM 2-mercaptoethanol). $\beta$-Gal activity assays were initiated $\left(\right.$ at $37^{\circ} \mathrm{C}$ ) by adding $0.2 \mathrm{ml}$ of o-nitrophenyl $\beta$ $D$-galactoside $(4 \mathrm{mg} / \mathrm{ml})$. The reaction mixtures were incubated with constant shaking at $37^{\circ} \mathrm{C}$ for $20 \mathrm{~min}$ and then terminated by the addition of $0.4 \mathrm{ml}$ of $1 \mathrm{M}$ $\mathrm{Na}_{2} \mathrm{CO}_{3}$. The reaction mixtures were centrifuged at $12,000 \times g$ for $2 \mathrm{~min}$, and the absorbance $\left(A_{420}\right)$ of the supernatants was determined. Relative $\beta$-gal activities were calculated from $A_{420}$ readings normalized to protein concentrations. Data were obtained from three independent experiments and averaged. Error bars indicate $( \pm 2 \times$ standard deviation).

\section{Reverse-transcription (RT)-PCR}

To determine the relative levels of specific lexA mRNAs derived from the fusion constructs, a semiquantitative RTPCR experiment was carried out following the protocols provided by the manufacturer (Invitrogen). Total RNA was first isolated from the transformants and then treated with DNase to remove contaminating DNA. Aliquots of RNA $(\sim 1 \mu \mathrm{g})$ were then reverse-transcribed into single-stranded complementary (c)DNA using an oligo-dT primer. After RNase $\mathrm{H}$ treatment, the single-stranded cDNA products were amplified by a PCR using a pair of specific primers. The forward and reverse primers respectively contained sequences complementary to nucleotides +1 to +21 (5'ATGAAAGCGTTAACGGCCAGG-3') and nucleotides +370 to +390 of lexA (5'-CAAGTCACCATCCATAATGCC-3'). As a control, the relative levels of actinspecific mRNAs in each preparation were also determined using a set of primers complementary to nucleotides +537 to +560 (5'-ACCAACTGGGACGATATGGAAAAG-3') and nucleotides +696 to +719 (5'-TTGGATGGAAACGTAGAAGGCTGG-3') of actin, respectively.

\section{Degradation assay}

To determine the turnover of the fusion proteins, analogous lex $A$ fusion constructs that were expressed under the control of an inducible GAL1 promoter were transformed into INVSc1. Transformants carrying these constructs were first grown in medium lacking histidine with $2 \%$ raffinose to a cell density of $\sim 1.0 A_{600}$ and then induced with $2 \%$ galactose for $1 \mathrm{~h}$. Afterward, cells were washed twice and then grown in medium containing $2 \%$ glucose and $100 \mu \mathrm{g} / \mathrm{ml}$ cycloheximide but lacking histidine. Cells were harvested at $0,0.5,1,2,4$, and $8 \mathrm{~h}$ postinduction and lysed. Forty-microgram samples of the cellular lysates were resolved on $10 \%$ polyacrylamide and electrophoresed at $100 \mathrm{~V}$ for $\sim 1 \mathrm{~h}$, and the proteins 
were transferred to a PVDF membrane and immunoblotted with an HRP-conjugated anti-LexA antibody.

\section{Acknowledgements}

This work was supported by a grant (NSC97-2311-B-008-003-MY3 to C.C.W.) from the National Science Council (Taipei, Taiwan). We wish to thank Prof. Shen-Liang Chen (National Central University, Jung-li, Taiwan) for helpful suggestions on the manuscript.

\section{Authors' contributions}

$\mathrm{CHL}$ generated the various lexA constructs and performed the RT-PCR, Western blotting, and transcriptional assays. GL and CPC performed the degradation and $\beta$-galactosidase assays. CCW coordinated the project and wrote the manuscript. All authors read and approved the final manuscript.

Received: 17 July 2010 Accepted: 16 November 2010

Published: 16 November 2010

\section{References}

1. Kuras L, Struhl K: Binding of TBP to promoters in vivo is stimulated by activators and requires Pol II holoenzyme. Nature 1999, 399(6736):609-613

2. Ptashne M, Gann A: Genes \& Signals. New York: USA: Cold Spring Harbor Laboratory Press; 2002

3. Sadowski I, Ma J, Triezenberg S, Ptashne M: GAL4-VP16 is an unusually potent transcriptional activator. Nature 1988, 335(6190):563-564.

4. Courey AJ, Holtzman DA, Jackson SP, Tjian R: Synergistic activation by the glutamine-rich domains of human transcription factor Sp1. Cell 1989, 59(5):827-836.

5. Mermod N, O'Neill EA, Kelly TJ, Tjian R: The proline-rich transcriptional activator of CTF/NF-I is distinct from the replication and DNA binding domain. Cell 1989, 58(4):741-753.

6. Green MR: Eukaryotic transcription activation: right on target. Mol Cell 2005, 18(4):399-402.

7. Traven A, Jelicic B, Sopta M: Yeast Gal4: a transcriptional paradigm revisited. EMBO Rep 2006, 7(5):496-499.

8. Wu Y, Reece RJ, Ptashne M: Quantitation of putative activator-target affinities predicts transcriptional activating potentials. EMBO J 1996, 15(15):3951-3963.

9. Carrozza MJ, John S, Sil AK, Hopper JE, Workman JL: Gal80 confers specificity on HAT complex interactions with activators. J Biol Chem 2002, 277(27):24648-24652.

10. Zenke FT, Engles R, Vollenbroich V, Meyer J, Hollenberg CP, Breunig KD: Activation of Gal4p by galactose-dependent interaction of galactokinase and Gal80p. Science 1996, 272(5268):1662-1665

11. Carter CW Jr: Cognition, mechanism, and evolutionary relationships in aminoacyl-tRNA synthetases. Annu Rev Biochem 1993, 62:715-748.

12. Martinis SA, Schimmel P: Escherichia coli and Salmonella Cellular and Molecular Biology. Am. Soc. Microbiol., Washington, DC. 21996.

13. Giege R, Sissler M, Florentz C: Universal rules and idiosyncratic features in tRNA identity. Nucleic Acids Res 1998, 26(22):5017-5035.

14. Pelchat M, Lapointe J: Aminoacyl-tRNA synthetase genes of Bacillus subtilis: organization and regulation. Biochem Cell Biol 1999, 77(4):343-347.

15. Maréchal-Drouard L, Weil JH, Dietrich A: Nuclear-encoded transfer RNAs in plant mitochondria. Annu Rev Cell Biol 1993, 8:115-131.

16. Tang HL, Yeh LS, Chen NK, Ripmaster T, Schimmel P, Wang CC: Translation of a yeast mitochondrial tRNA synthetase initiated at redundant nonAUG codons. J Biol Chem 2004, 279(48):49656-49663.

17. Chang KJ, Wang CC: Translation initiation from a naturally occurring nonAUG codon in Saccharomyces cerevisiae. J Biol Chem 2004, 279(14):13778-13785

18. Natsoulis G, Hilger F, Fink GR: The HTS1 gene encodes both the cytoplasmic and mitochondrial histidine tRNA synthetases of $S$. cerevisiae. Cell 1986, 46(2):235-243.

19. Chatton B, Walter P, Ebel JP, Lacroute F, Fasiolo F: The yeast VAS1 gene encodes both mitochondrial and cytoplasmic valyl-tRNA synthetases. $J$ Biol Chem 1988, 263(1):52-57.
20. Mirande M: Aminoacyl-tRNA synthetase family from prokaryotes and eukaryotes: structural domains and their implications. Prog Nucleic Acid Res Mol Biol 1991, 40:95-142.

21. Wang CC, Schimmel P: Species barrier to RNA recognition overcome with nonspecific RNA binding domains. J Biol Chem 1999, 274(23):16508-16512.

22. Wang CC, Morales AJ, Schimmel P: Functional redundancy in the nonspecific RNA binding domain of a class I tRNA synthetase. J Biol Chem 2000, 275(22):17180-17186.

23. Kaminska $M$, Deniziak $M$, Kerjan $P$, Barciszewski J, Mirande $M$ : A recurrent general RNA binding domain appended to plant methionyl-tRNA synthetase acts as a cis-acting cofactor for aminoacylation. EMBO J 2000, 19(24):6908-6917.

24. Francin $M$, Kaminska $M$, Kerjan $P$, Mirande $M$ : The $N$-terminal domain of mammalian Lysyl-tRNA synthetase is a functional tRNA-binding domain. $J$ Biol Chem 2002, 277(3):1762-1769.

25. Simos G, Segref A, Fasiolo F, Hellmuth K, Shevchenko A, Mann M, Hurt EC: The yeast protein Arc1p binds to tRNA and functions as a cofactor for the methionyl- and glutamyl-tRNA synthetases. EMBO J 1996, 15(19):5437-5448.

26. Negrutskii BS, Shalak VF, Kerjan P, El'skaya AV, Mirande M: Functional interaction of mammalian valyl-tRNA synthetase with elongation factor EF-1alpha in the complex with EF-1H. J Biol Chem 1999, 274(8):4545-4550.

27. Schimmel P, Wang CC: Getting tRNA synthetases into the nucleus. Trends Biochem Sci 1999, 24(4):127-128.

28. Martinis SA, Plateau P, Cavarelli J, Florentz C: Aminoacyl-tRNA synthetases: a family of expanding functions. Mittelwihr, France, October 10-15, 1999. EMBO J 1999, 18(17):4591-4596.

29. Francklyn C, Perona JJ, Puetz J, Hou YM: Aminoacyl-tRNA synthetases: versatile players in the changing theater of translation. RNA 2002 8(11):1363-1372

30. Chang CP, Lin G, Chen SJ, Chiu WC, Chen WH, Wang CC: Promoting the formation of an active synthetase/tRNA complex by a nonspecific tRNAbinding domain. J Biol Chem 2008, 283(45):30699-30706.

31. Chiu WC, Chang CP, Wang CC: Evolutionary basis of converting a bacterial tRNA synthetase into a yeast cytoplasmic or mitochondrial enzyme. J Biol Chem 2009, 284(36):23954-23960.

doi:10.1186/1471-2199-11-85

Cite this article as: Lin et al:: A tryptophan-rich peptide acts as a transcription activation domain. BMC Molecular Biology 2010 11:85.

\section{Submit your next manuscript to BioMed Central and take full advantage of:}

- Convenient online submission

- Thorough peer review

- No space constraints or color figure charges

- Immediate publication on acceptance

- Inclusion in PubMed, CAS, Scopus and Google Scholar

- Research which is freely available for redistribution 